\title{
Cincinnati Prehospital Stroke Scale for EMS Redirection of Large Vessel Occlusion Stroke
}

\author{
Ahmad Nehme (D), Yan Deschaintre, Marilyn Labrie, Nicole Daneault, \\ Céline Odier, Alexandre Y. Poppe, Dave Ross, Christian Stapf, \\ Grégory Jacquin, Laura C. Gioia
}

\begin{abstract}
Introduction: Prehospital identification of large vessel occlusion (LVO) stroke may expedite treatment by direct transport to comprehensive stroke centers (CSCs) with endovascular capabilities. The Cincinnati Prehospital Stroke Scale (CPSS) is commonly used for prehospital stroke detection. We aimed to assess whether (1) a high CPSS score can identify LVO and (2) an Emergency Medical Service (EMS) redirection protocol based on high CPSS accelerated endovascular treatment (EVT). Methods: A retrospective comparison of patients transported by EMSs for suspected stroke to a high-volume CSC over a 16-month period, before and after implementation of an EMS redirection protocol based on high CPSS score (3/3). Charts were reviewed to determine the presence of LVO. Time to EVT and 3-month outcomes were compared before and after implementation. Results: A prehospital CPSS 3/3 score was found in $223(59 \%)$ patients, demonstrating positive and negative predictive values for LVO of $29 \%$ and $94 \%$, respectively. CPSS-based EMS redirection increased the proportion of EVT performed after direct transport to CSC [before: 21 (36\%), after: 45 (63\%), $p<0.01$ ] and decreased median first door-to-groin puncture time by 28 minutes [109 (interquartile range (IQR) 64-116) versus 81 (IQR 56-130), $p=0.03$ ]. At 3 months, the proportion of patients achieving functional independence (modified Rankin score $0-2$ ) went from 20/57 (35\%) to 29/68 (43\%) $(p=0.39)$ following implementation. Conclusions: CPSS-based EMS redirection accelerated identification of LVO strokes in the out-of-hospital setting and decreased time to EVT. Nevertheless, this protocol was also associated with high rates of non-LVO stroke. Impact on clinical outcomes should be evaluated in a larger cohort.
\end{abstract}

RÉSUMÉ: Utilité de l'échelle de Cincinnati pour la redirection des occlusions artérielles cérébrales proximales par les services médicaux d'urgence. Introduction : Dans un contexte pré-hospitalier, le fait de pouvoir identifier l'occlusion de vaisseaux sanguins cérébraux proximaux peut accélérer l'amorce d'un traitement en favorisant un transfert direct vers un centre complet de prise en charge des AVC (comprehensive stroke centers) doté de moyens d'intervention endovasculaire. L'échelle de Cincinnati (Cincinnati Prehospital Stroke Scale ou CPSS) est couramment utilisée afin de détecter les signes d'un AVC dans un contexte pré-hospitalier. Notre intention est double ici : 1) évaluer dans quelle mesure un score élevé à la CPSS peut permettre d'identifier l'occlusion de vaisseaux sanguins cérébraux proximaux ;2) évaluer dans quelle mesure un protocole des services médicaux d'urgence consistant à rediriger des patients en fonction d'un score élevé à la CPSS a permis d'accélérer l'offre d'un traitement endovasculaire. Méthodes : Au cours d'une période de 16 mois, soit avant et après la mise sur pied de ce protocole en fonction d'un score élevé à la CPSS (3/3), nous avons effectué une comparaison rétrospective des dossiers de patients transportés vers des centres complets de prise en charge des AVC en raison de soupçons d'AVC. Ces dossiers ont été analysés afin de pouvoir identifier les cas d'occlusion proximales. Enfin, les délais permettant d'obtenir un traitement endovasculaire et l'évolution de l'état de santé des patients au bout de 3 mois ont été comparés avant et après la mise sur pied de ce protocole. Résultats : En fonction d'un score de 3/3 à la CPSS obtenu en contexte pré-hospitalier a été observé chez 223 patients (59\%), la valeur prédictive positive et négative pour des cas d'occlusion de vaisseaux sanguins cérébraux proximaux étant respectivement de $29 \%$ et de $94 \%$. Le fait que les services médicaux d'urgence redirigent des patients en fonction d'un certain score à la CPSS a fini par augmenter le nombre de traitements endovasculaires prodigués à la suite d'un transfert direct vers un centre complet de prise en charge des AVC (avant le protocole : 21 (36\%); après le protocole : $45(63 \%) ; p<0,01)$ et par diminuer de 28 minutes les délais entre la prise en charge à l'arrivée et la ponction artérielle (109 [intervalle interquartile de 64-116] contre 81 [intervalle interquartile de 56-130] ; $\mathrm{p}=0,03$ ). Au bout de 3 mois, à la suite de la mise sur pied du protocole, la proportion de patients autonomes sur le plan fonctionnel (échelle de Rankin modifiée : 0-2) est passée de 20/57 (35\%) à 29/68 (43\%) (p=0,39). Conclusion : Le fait de rediriger des patients en fonction d'un certain score à la CPSS a permis d'accélérer la détection de cas d'AVC survenus à la suite de l'occlusion de vaisseaux sanguins cérébraux proximaux et donc de réduire les temps d'intervention. Cela dit, ce protocole a aussi été associé à un taux élevé de détection d'AVC non produits par l'occlusion de vaisseaux sanguins cérébraux proximaux. L'impact de cette constatation en ce qui regarde l'évolution de l'état de santé des patients devrait être évalué dans une plus vaste cohorte.

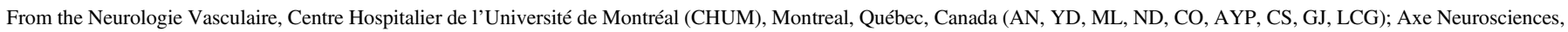

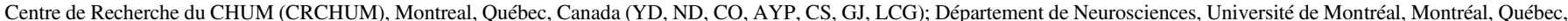

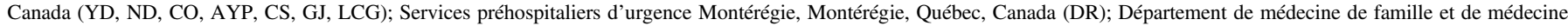
d'urgence, Université de Montréal, Montreal, Québec, Canada (DR)

Received May 29, 2019. Final Revisions Submitted June 24, 2019. Date of Acceptance July 6, 2019

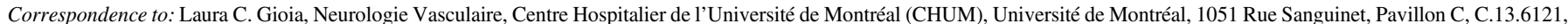
Montréal, Québec H2X 0C1, Canada. Email: laura.gioia@umontreal.ca 


\section{INTRODUCTION}

Multiple prehospital scales have been developed to facilitate prehospital identification of acute stroke by Emergency Medical Service (EMS) providers. This strategy has increased the number of patients eligible for intravenous thrombolysis and acclerated treatment times. ${ }^{1}$ In recent years, multiple clinical trials have established endovascular treatment (EVT) as an effective therapy in acute ischemic stroke due to intracranial large vessel occlusion (LVO) ${ }^{2-6}$ Access to EVT remains, however, limited due to the restricted time window for intervention and accessibility to comprehensive stroke centers (CSCs) with endovascular capabilities. ${ }^{7}$ As such, the need to expedite EVT for acute stroke has imposed a restructuring of prehospital stroke care, with emphasis on accurately identifying LVO in the prehospital setting. ${ }^{8}$

Multiple LVO-specific stroke scales [e.g. Rapid Arterial oCclusion Evaluation (RACE) and Cincinnati Stroke Triage Assessment Tool] have been developed to increase the detection of LVO in the prehospital setting. ${ }^{9,10}$ Implementation of a new LVO-specific scale might not always be feasible given the resources required (time and cost of retraining EMS personnel and situations where multiple EMS agencies serve one territory). The Cincinnati Prehospital Stroke Scale (CPSS) is simple and made up of three clinical items (facial droop, arm drift, and abnormal speech) with high sensitivity (79-95\%), moderate specificity (24-79\%), and good interobserver reliability (correlation coefficient 0.89 ) for ischemic stroke diagnosis. ${ }^{11-13}$ In an observational retrospective in-hospital study, a CPSS score of $3 / 3$ had a $32 \%$ positive and $90 \%$ negative predictive value for LVO. ${ }^{14}$ The use of a high CPSS score could potentially detect LVO in the prehospital setting, yet this has not been studied in an unselected cohort of suspected stroke patients.

The overall aims of this study were to (1) evaluate the ability of a high CPSS score to identify LVO stroke in unselected patients with acute prehospital neurological symptoms and (2) determine the effect of this strategy on patient volume, time to treatment, and clinical outcomes. We hypothesized that the use of a high CPSS score as an EMS redirection tool would increase the detection of LVO stroke in the prehospital setting, accelerate time to EVT, and improve rates of functional independence at 90 days.

\section{Methods}

\section{Study Design}

We conducted a retrospective observational study of all consecutive patients transported by EMS to the Emergency Department of the Centre Hospitalier de l'Université de Montréal (CHUM) during the study period. Data were collected between February 2016 and June 2017, that is, two 8-month periods, before and after implementation of a city-wide CPSS-based EMS redirection protocol for suspected LVO stroke directly to one of the two CSCs in Montreal.

\section{EMS Service and Suspected Stroke}

Emergency prehospital care in the city of Montreal, Canada (population 2.3 million) is coordinated by a single public-tiered response EMS agency that employs first responders and primary care paramedics (>900). In the out-of-hospital setting, EMS providers currently employ the CPSS scale for acute stroke detection. Once acute stroke is suspected (CPSS $\geq 1$ ), EMS providers call a centralized paramedic to determine patient orientation according to geolocation. Prior to the EMS redirection protocol, all acute stroke patients were transferred to the nearest stroke center [either primary stroke center (PSC) or CSC].

In 2016, a modified use of the CPSS scale was proposed, whereby a high CPSS score [i.e. positive for all three items (3/3)] would serve as a method to detect LVO in the prehospital setting, thereby minimizing resources required for the implementation of a new stroke severity scale. Accordingly, any patient with a high CPSS score (3/3) and a time from symptom onset/last time seen well $<5$ hours was directly transported to the nearest CSC, thereby bypassing PSC. The EMS redirection protocol was implemented in October 2016. The CHUM is situated on average at a $20 \mathrm{~km}$ distance from the three PSCs to which it is affiliated and that were affected by the redirection protocol.

\section{Study Population and Data Collection}

All patients first evaluated at the CSC for suspected acute stroke were identified via an electronic patient record system used for acute stroke evaluations. This list was cross-referenced with a centralized registry kept at EMS headquarters of all consecutive suspected stroke patients redirected to CSC. For patients with a documented CPSS, hospital charts and neuroimaging were reviewed to determine initial prehospital CPSS score, final diagnosis, presence of LVO, and use of reperfusion therapy (thrombolysis/EVT). Final diagnosis was categorized as LVO stroke, non-LVO stroke, transient ischemic attack (TIA), intracerebral hemorrhage (ICH), or stroke mimic. LVO was determined on initial vascular imaging (cerebral angio-CT or direct cerebral angiography) and defined as an occlusion of the terminal intracranial carotid artery, proximal middle cerebral artery (M1 segment), tandem (extracranial carotid artery plus M1), or basilar artery.

For analysis of time to EVT and 3-month clinical outcomes, patients who underwent EVT (after direct transport to CSC or after secondary transfer from the three PSCs affected by the redirection protocol) were identified in a prospective database of all thrombectomies performed at the CHUM. Patients treated by EVT within the study period were compared before and after implementation of the CPSS-based redirection protocol and were further stratified based on the location of first medical evaluation (CSC or PSC).

In the periods before and after implementation, first door-togroin puncture (DTP) times were calculated from the time of first 


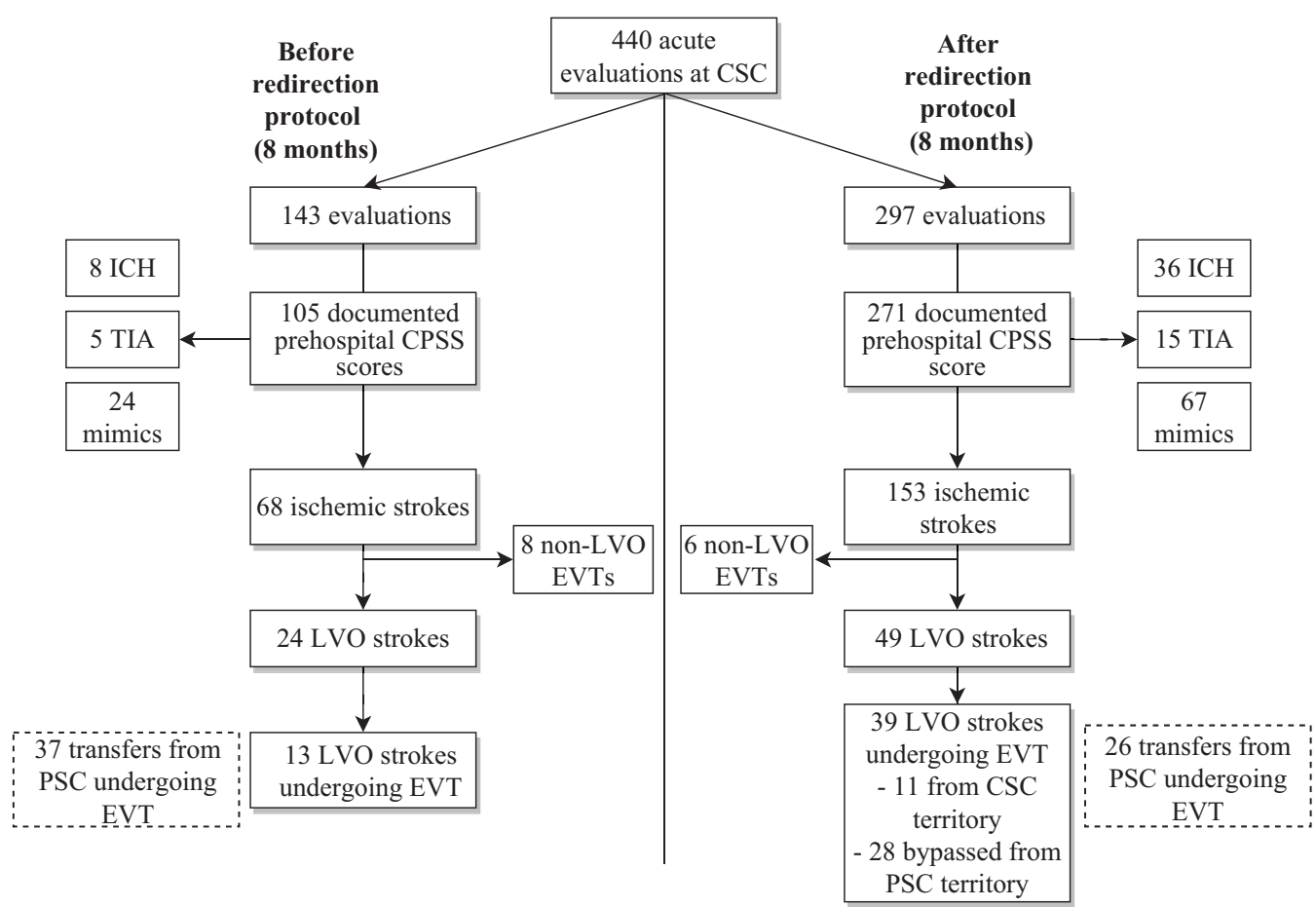

Figure 1: Patients evaluated at the comprehensive stroke center (CSC) before and after implementation of CPSSbased EMS redirection protocol. CPSS: Cincinnati Prehospital Stroke Scale score; EVT: endovascular therapy; LVO: large vessel occlusion; PSC: primary stroke center.

medical contact (at either PSC or CSC) to groin puncture at CSC. EMS-to-first medical contact and door-to-needle (DTN) times were also assessed. Modified Rankin scores (mRSs) were determined at 3-month clinical follow-up or by telephone interview as part of existing quality assurance procedures for all EVT cases.

All non-LVO stroke patients receiving only intravenous thrombolysis during the study period were also identified. Three-month functional outcomes were determined in the periods before and after implementation of redirection protocol. Moreover, in the post-implementation period, EMS to needle time was compared between patients transported from CSC territory in contrast to redirected from PSC areas.

\section{Statistics}

Continuous variables are reported as means and standard deviations (SDs) or median and interquartile ranges (IQRs), as appropriate. Dichotomous variables are reported as proportions. Student's $t$-test or Mann-Whitney $U$ tests were used to compare parametric and nonparametric variables, respectively. Dichotomous variables were compared by Pearson's chi-squared or Fisher's exact test. Clinical outcomes were dichotomized as favorable (mRS 0-2) or unfavorable (mRS 3-6) and compared using Pearson's chi-squared test. A $p$-value cut-off of 0.05 was established for statistical significance. SPSS 25 was used for statistical analyses.

\section{Standard Protocol Approvals, Registrations, Patient Consent}

This study was approved for waiver of consent by the ethics committee at the CHUM.

\section{Results \\ Patient Populations before and after EMS Redirection Protocol}

During the study period, 440 patients with suspected acute stroke were transported by EMS directly to CSC (Figure 1). The EMS redirection protocol led to a 2.1-fold increase of direct transports to CSC by EMS [143 (33\%) before versus 297 (67\%) after]. Of these, 376 patients had a CPSS score documented by EMS [105 (73\%) before compared to 271 (91\%) after].

Most patients with a documented CPSS score were diagnosed with an ischemic stroke [before: 68 (65\%); after: 153 (57\%), $p=0.14]$. While EMS redirection led to a two-fold increase in the absolute number of patients with LVO stroke first evaluated at CSC, the proportion of LVO strokes remained similar between time periods [before: 24 (23\%); after: 49 (18\%), $p=0.29$ ]. Following implementation, among patients with a documented CPSS score, the proportion of ICH slightly increased [8 (8\%) versus 36 (13\%), $p=0.13$ ], stroke mimic rates remained similar [24 (23\%) versus $67(25 \%), p=0.70]$, and less ischemic stroke patients received no recanalization therapy (neither thrombolysis nor EVT) [34 (32\%) versus $58(21 \%), p=0.03$ ]. Furthermore, among all patients undergoing EVT, the proportion transferred to CSC following PSC evaluation significantly decreased from $37 / 58$ $(64 \%)$ to $26 / 71(37 \%)$ after implementation $(p<0.01)$. A total of six patients were transferred from PSC for EVT but did not undergo intervention (four before and two after implementation of redirection protocol). The reasons for exclusion from EVT were improvement of neurological status in four, overestimated ASPECT score in one, and delay leading to deterioration of ASPECT score in 
Table 1: Performance of the CPSS score for LVO identification in patients first evaluated at CSC

\begin{tabular}{c|c|c|c|c|c|c}
\hline CPSS score & LVO stroke & Non-LVO stroke & ICH & TIA & Stroke mimic & Total \\
\hline $3(n$, row $\%)$ & $64(29)$ & $88(39)$ & $35(16)$ & $7(3)$ & $29(13)$ & 223 \\
\hline 2 & $6(9)$ & $29(45)$ & $4(6)$ & $3(5)$ & $23(35)$ & 65 \\
\hline 1 & $3(5)$ & $24(36)$ & $3(5)$ & $6(9)$ & $30(45)$ & 66 \\
\hline 0 & 0 & $7(32)$ & $2(9)$ & $4(18)$ & $9(41)$ & 22 \\
\hline
\end{tabular}

Table 2: Baseline patient characteristics undergoing EVT before and after implementation of EMS redirection protocol

\begin{tabular}{|c|c|c|c|c|c|c|c|c|}
\hline & \multicolumn{3}{|c|}{ Before } & \multicolumn{4}{|c|}{ After } & \multirow[b]{2}{*}{$p$-value } \\
\hline & $\begin{array}{l}\text { Direct transfer to } \\
\text { CSC }(n=21)\end{array}$ & $\begin{array}{l}\text { Secondary transfer } \\
\text { from PSC }(n=37)\end{array}$ & $\begin{array}{l}\text { Total patients } \\
\quad(n=58)\end{array}$ & $\begin{array}{l}\text { Direct transfer to } \\
\quad \text { CSC (CSC } \\
\text { territory) }(n=16)\end{array}$ & $\begin{array}{l}\text { Direct transfer to } \\
\text { CSC (redirected } \\
\text { from PSC } \\
\text { territory) }(n=29)\end{array}$ & $\begin{array}{l}\text { Secondary transfer } \\
\text { from PSC }(n=26)\end{array}$ & $\begin{array}{l}\text { Total patients } \\
\quad(n=71)\end{array}$ & \\
\hline $\begin{array}{l}\text { Age, years (mean } \\
\pm \mathrm{SD})\end{array}$ & $76 \pm 13$ & $70 \pm 16$ & $72 \pm 15$ & $72 \pm 14$ & $73 \pm 15$ & $75 \pm 14$ & $73 \pm 14$ & 0.65 \\
\hline Women, $n(\%)$ & $12(57)$ & $17(46)$ & $29(50)$ & 7 (44) & $11(38)$ & $18(69)$ & $36(51)$ & 0.94 \\
\hline $\begin{array}{l}\text { NIHSS, median } \\
\text { (IQR) }\end{array}$ & $20(16-24)$ & $17(11-23)$ & $17(14-23)$ & $18(17-21)$ & $16(13-21)$ & $17(13-21)$ & $17(14-21)$ & 0.56 \\
\hline $\begin{array}{l}\text { ASPECT, median } \\
\text { (IQR) }\end{array}$ & $9(8-10)$ & $9(8-10)$ & $9(8-10)$ & $10(8-10)$ & $10(8-10)$ & $9(7-10)$ & $9(8-10)$ & 0.57 \\
\hline tPA use, $n(\%)$ & $16(76)$ & $28(76)$ & $44(76)$ & $9(56)$ & $26(90)$ & $17(65)$ & $52(73)$ & 0.73 \\
\hline \multicolumn{9}{|c|}{ Occlusion site } \\
\hline $\begin{array}{l}\text { Extracranial } \\
\text { carotid, } n(\%)\end{array}$ & 0 & $1(3)$ & $1(2)$ & 0 & 0 & 0 & 0 & \\
\hline $\begin{array}{l}\text { Tandem } \\
\text { occlusion }\end{array}$ & $3(14)$ & 7 (19) & $10(17)$ & 0 & $3(11)$ & $4(15)$ & $7(10)$ & 0.22 \\
\hline $\begin{array}{l}\text { Intracranial } \\
\text { carotid }\end{array}$ & $6(29)$ & $5(13)$ & $11(19)$ & $6(38)$ & $4(14)$ & 0 & $10(14)$ & 0.46 \\
\hline M1 & $8(38)$ & $17(46)$ & $25(43)$ & $5(31)$ & $20(69)$ & $18(69)$ & $43(61)$ & 0.05 \\
\hline M2 & $3(14)$ & $6(16)$ & $9(16)$ & $5(31)$ & $1(3)$ & $3(12)$ & $9(12)$ & 0.64 \\
\hline Basilar & $1(5)$ & $1(3)$ & $2(3)$ & 0 & $1(3)$ & $1(4)$ & $2(3)$ & 0.84 \\
\hline \multicolumn{9}{|c|}{ Recanalization } \\
\hline TICI $2 \mathrm{~B} / 3, n(\%)$ & $14(67)$ & $25(68)$ & $39(67)$ & $6(37.5)$ & $24(83)$ & $23(88)$ & $53(74)$ & 0.35 \\
\hline TICI 2A & $4(19)$ & $8(22)$ & $12(21)$ & $4(25)$ & $2(7)$ & $3(12)$ & $9(13)$ & 0.22 \\
\hline TICI 0-1 & $3(14)$ & $4(10)$ & 7 (12) & $6(37.5)$ & $3(10)$ & 0 & $9(13)$ & 0.92 \\
\hline
\end{tabular}

Comparisons are between total groups before and after implementation of CPSS-based EMS redirection.

one. Among all patients receiving thrombolytic therapy, median (IQR) DTN was similar before [39 (30-48) minutes] and after implementation [36 (28-44) minutes] $(p=0.15)$.

\section{Use of CPSS 3/3 for Prehospital LVO Detection}

Most patients first evaluated at CSC had a CPSS 3/3 score [223 (59\%)] and the number of patients with CPSS 3/3 increased from $42(40 \%)$ to $181(67 \%)$ after implementation of the EMS redirection protocol $(p<0.01)$. Among all patients with CPSS $3 / 3$, the final diagnosis was LVO stroke in $64(29 \%)$, non-LVO stroke in 88 (39\%), TIA in 7 (3\%), ICH in $35(16 \%)$, and stroke mimic in 29 (13\%) (Table 1). A prehospital CPSS score of 3/3 demonstrated a $29 \%$ PPV and a 94\% NPV for LVO detection.
Comparison of Time to EVT and 3-Month Outcomes before and after Implementation

A total of 129 patients underwent EVT during the study period (58 patients before and 71 patients after redirection implementation) (Table 2). Baseline demographic characteristics and stroke severity did not differ between groups, although there was a slightly higher proportion of M1 occlusions following implementation [25 (43\%) versus $43(61 \%)$, respectively, $p=0.05$ ]. Similarly, rates of TICI 2B/3 flow were slightly higher following implementation of the redirection protocol [before: $39(67 \%)$; after: $53(74 \%), p=0.35]$.

Out-of-hospital transport time [defined as median (IQR) time from EMS arrival to stroke center arrival] slightly increased from 30 (IQR 22-34) to 34 (IQR 28-43) minutes after implementation 
Table 3: Time to treatment before and after implementation of redirection protocol for all patients undergoing EVT

\begin{tabular}{|c|c|c|c|c|c|c|c|c|}
\hline & \multicolumn{3}{|c|}{ Before } & \multicolumn{4}{|c|}{ After } & \multirow[b]{2}{*}{$p$-value } \\
\hline & $\begin{array}{l}\text { Direct from CSC } \\
\text { territory }(n=21)\end{array}$ & $\begin{array}{l}\text { Transfer after PSC } \\
\text { evaluation }(n=37)\end{array}$ & Total $(n=58)$ & $\begin{array}{l}\text { Direct from CSC } \\
\text { territory }(n=16)\end{array}$ & $\begin{array}{l}\text { Bypassed to CSC } \\
\text { from PSC territory } \\
\quad(n=29)\end{array}$ & $\begin{array}{l}\text { Transfer after PSC } \\
\text { evaluation }(n=26)\end{array}$ & Total $(n=71)$ & \\
\hline $\begin{array}{l}\text { EMS to door, } \\
\text { minutes, } \\
\text { median (IQR) }\end{array}$ & $30(21-33)$ & $30(23-36)$ & $30(22-34)$ & $31(22-36)$ & $37(29-44)$ & $35(32-40)$ & $34(28-43)$ & 0.03 \\
\hline $\begin{array}{r}\text { Door-to-needle, } \\
\text { median (IQR) }\end{array}$ & $34(30-43)$ & $53(39-69)$ & $43(34-64)$ & $37(26-41)$ & $32(27-40)$ & $55(44-76)$ & $37(28-51)$ & 0.06 \\
\hline $\begin{array}{l}\text { Door-to-groin } \\
\text { puncture, } \\
\text { median (IQR) }\end{array}$ & $61(49-67)$ & $139(112-163)$ & $109(64-146)$ & $65(57-80)$ & $58(44-77)$ & $145(122-167)$ & $81(56-130)$ & 0.03 \\
\hline
\end{tabular}

Comparisons are between total groups before and after implementation of CPSS-based EMS redirection.
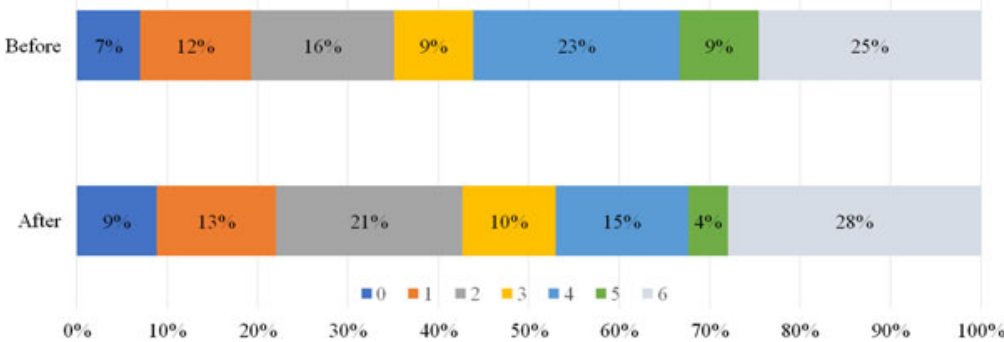

Figure 2: (Colour online) Three-month modified Rankin scores (mRSs) following endovascular treatment (EVT) before and after implementation of CPSS-based EMS redirection protocol. All patients planned for EVT with documented mRSs are included. Clinical outcomes were dichotomized as favorable (mRS 0-2) or unfavorable (3-6).

( +4 minutes), $p=0.03$ (Table 3 ). In the period following implementation of the redirection protocol, median out-of-hospital time (EMS to CSC time) was 6 minutes longer for patients who were within the PSC territory but redirected to CSC when compared to those patients transported from within the CSC territory [37 (IQR 29-44) versus 31 (IQR 22-36), respectively, $p=0.40]$. Despite slight transport delays, overall median DTN time for thrombolytic therapy decreased by 6 minutes following implementation [43 (IQR 34-64) versus 37 (IQR 28-51), $p=0.06$ ]. Similarly, overall median first DTP time decreased by 28 minutes following EMS redirection protocol in all patients [109 (IQR 64-146) versus 81 (IQR 56-130) minutes, $p=0.03$ ]. The decrease in DTP was more pronounced when comparing the subgroup of patients directly affected by the redirection protocol [median DTP of 139 (112-163) minutes in patients transferred to CSC from PSC prior to implementation compared to 58 (44-77) minutes in patients bypassed to CSC from PSC territory: median difference of 81 minutes].

Three-month clinical outcomes were available for 125/129 $(97 \%)$ patients who underwent EVT during the study period. The proportion of patients achieving functional independence (defined as mRS 0-2) was similar overall [20/57 (35\%) before and 29/68 (43\%) after implementation, $p=0.39$ ] but trended toward higher rates following redirection protocol (Figure 2).

\section{Effect of Redirection Protocol on Non-LVO Stroke}

Following implementation, a total of 56 stroke patients received IV thrombolysis without EVT after direct transfer to CSC. Of these, 16/56 (29\%) were transported from CSC territory and 40/56 $(71 \%)$ were redirected from PSC areas. Median (IQR) EMS to needle times were similar between the two groups: 70 (61-88) minutes for patients from CSC territory and 70 (63-84) minutes for patients redirected from PSC areas $(p=0.90)$. During the entire study period, 77 stroke patients were treated with IV thrombolysis without EVT. Three-month mRSs were available in 72/77 (94\%) patients. Rates of functional independence (mRS 0-2) remained similar despite implementation of the redirection protocol $[9 / 21$ (43\%) before compared to $22 / 51(43 \%)$ after, $p=0.98]$.

\section{Discussion}

Implementation of an EMS redirection protocol based on a high CPSS score (3/3) accelerated identification of LVO stroke in the out-of-hospital setting. On the other hand, utilization of a high CPSS score as an LVO triage tool was also associated with high patient volume and high rates of non-LVO ischemic stroke and $\mathrm{ICH}$, resulting in an overall low PPV for LVO detection. These findings are similar to other studies assessing the predictive value of high CPSS score for LVO detection, although these studies 
were based on selective cohorts of confirmed ischemic stroke or following in-hospital evaluations. ${ }^{14,15}$

Repurposing the CPSS scale for prehospital LVO detection is appealing given its simplicity and well-established use by EMS providers for acute stroke detection. While several prehospital stroke severity scales have been developed (which include clinical signs predictive for LVO such as gaze deviation and spatial neglect), the capacity to implement these scales and their ease of administration by EMS providers are questionable. ${ }^{9,10}$ Indeed, while a high RACE score was found to have a higher PPV (42\%) for LVO detection in a prospective cohort of 357 patients, RACE scores were not completed in $60 \%$ of patients transported by EMS. ${ }^{9}$ In our study, CPSS scores were available in $86 \%$ of patients, given its routine widespread use by EMS providers for prehospital stroke identification. Furthermore, modified use of the CPSS scale as a triage tool for LVO detection was easy to implement and required minimal training. Such a strategy may be an interesting alternative to accelerate detection of prehospital EVT candidates in systems of care unable to implement a more complex LVO detection scale.

On the other hand, although a high CPSS score detected the majority of LVO strokes in our study, its use as a redirection tool also led to a substantial increase in patient volume, with a minority of patients ultimately being LVO strokes treated by EVT. Indeed, the EMS redirection protocol doubled the number of EMS transports and stroke team mobilizations over a brief time period, translating to approximately 20 supplementary evaluations per month. This increase might not be possible to absorb in certain stroke systems with limited resources. Nonetheless, bypassing PSC significantly accelerated overall median time to EVT, with thrombectomy initiated approximately 80 minutes faster for the individual patient after direct transport to CSC compared to prior secondary transfer after PSC evaluation. Furthermore, given that a significant proportion of patients with severe ischemic stroke or ICH are ultimately transferred to higher levels of care, EMS redirection of a proportion of non-LVO patients might be CSC-appropriate, particularly in systems where CSCs can absorb the increased workload (e.g. systems with less developed PSCs and/or concentration of resources at CSCs).

The optimal prehospital scale may not be uniform and might vary by region/geography and distribution of stroke centers. The optimal trade-off acceptable between high PPV and high NPV may differ based on the distances between PSC and CSC. In regions with a large distance between PSC and CSC, prehospital scales with higher PPV for LVO detection may be ideal, as to not delay thrombolysis in non-LVO stroke. On the contrary, in regions where PSC and CSC are in close proximity (e.g. urban settings such as Montreal), less specific prehospital scales like the CPSS could expedite EVT treatment in LVO stroke, without delaying and possibly accelerating thrombolytic therapy in nonLVO stroke. The difference in DTN times between PSC and CSC should also be considered, as increases in transport time may be offset by faster access to thrombolysis in CSCs with established protocols.

EMS redirection using the CPSS decreased time metrics in acute LVO stroke. Our findings are in line with a recent Danish study showing that EMS redirection of LVO stroke led to a decrease in time to EVT without significantly increasing DTN times, albeit using a different EMS redirection strategy. ${ }^{16}$ The Danish study, however, was conducted on a significantly larger territory $\left(13,000 \mathrm{~km}^{2}\right.$ versus approximately $500 \mathrm{~km}^{2}$ in our study), with greater distance between PSC and CSC (150 versus $20 \mathrm{~km}$ ), and its results are thus not applicable in a densely populated urban setting. Moreover, our study confirms that EMS redirection of suspected LVO stroke not only significantly decreases time to EVT but also appears to be associated with faster administration of thrombolysis in patients bypassed to CSC also undergoing EVT. Clinical outcomes at 3 months were similar for non-LVO stroke patients receiving thrombolysis without EVT, likely because increase in transport time is offset by faster access to thrombolysis at CSC versus PSC.

Our findings also suggest that EMS redirection of LVO stroke might be associated with a higher proportion of functional independence at 3 months. A recent study showed that with every minute saved before EVT, an extra four quality-adjusted life days are achieved. ${ }^{17}$ Extrapolating those results to our findings, the approximate 80-minute decrease in EMS to groin time for the individual patient bypassing PSC to reach CSC could translate, on average, in nearly a full year of extra healthy life. However, given that these results are based on a small sample, they are hypothesis-generating only. These findings support current ongoing randomized clinical trials evaluating EMS redirection to CSC compared to transfer of established LVO strokes from PSC.

Limitations of this study include its retrospective analysis of a single high-volume urban CSC, thereby limiting generalizability to other centers and to patients redirected from further rural areas. Data from the second CSC in our city were not available, and results may not be generalizable on a city-wide level. City-wide data of all patients transferred to PSC for acute stroke (CPSS 0-2/3) were not available to permit analysis of the overall sensitivity and specificity of CPSS $3 / 3$ for LVO detection. The brief period over which it was conducted not only limited its sample size but also controlled for bias from improved overall care of LVO stroke over time, although TICI $2 \mathrm{~B} / 3$ rates were slightly higher following implementation of the redirection protocol.

\section{Conclusion}

Although CPSS-based EMS redirection did detect the majority of LVO strokes, it did not increase the rate of LVO strokes directly transported to CSC on account of a substantial rise in non-LVO patient volume. Nevertheless, this strategy decreased time to reperfusion therapy for patients amenable to EVT, without introducing significant treatment delays in non-LVO stroke patients undergoing thrombolysis. Repurposing the CPSS scale for prehospital LVO stroke detection may improve clinical outcomes, but larger studies are warranted comparing the CPSS to more complex yet potentially more specific LVO stroke detection scales.

\section{DisclosuRES}

AN reports no disclosures. YD reports personal fees from Servier, outside the submitted work. ML reports no disclosures. ND reports no disclosures. CO reports no disclosures. AYP reports no disclosures. DR reports no disclosures. CS reports no disclosures. GJ reports no disclosures. LCG reports grants and personal fees from Servier, personal fees from Bayer, personal fees from Pfizer, personal fees from BMS, outside the submitted work. 


\section{Statement of Authorship}

AN designed and conceptualized the study, collected data, performed analysis and interpretation of the data, and drafted the manuscript. YD provided data and revised the manuscript. ML provided data and revised the manuscript. ND provided data and revised the manuscript. $\mathrm{CO}$ provided data and revised the manuscript. AYP provided data and revised the manuscript. DR provided data and revised the manuscript. CS provided data and revised the manuscript. GJ collected data, performed analysis and interpretation of the data, and revised the manuscript. LCG designed and conceptualized the study, collected data, performed analysis and interpretation of the data, and revised the manuscript.

\section{REFERENCES}

1. Bae HJ, Kim DH, Yoo NT, et al. Prehospital notification from the emergency medical service reduces the transfer and intra-hospital processing times for acute stroke patients. J Clin Neurol. 2010;6(3):138-42.

2. Berkhemer OA, Fransen PS, Beumer D, et al. A randomized trial of intraarterial treatment for acute ischemic stroke. N Engl J Med. 2015;372(1):11-20.

3. Goyal M, Demchuk AM, Menon BK, et al. Randomized assessment of rapid endovascular treatment of ischemic stroke. N Engl J Med. 2015;372(11):1019-30.

4. Saver JL, Goyal M, Bonafe A, et al. Stent-retriever thrombectomy after intravenous t-PA vs. t-PA alone in stroke. N Engl J Med. 2015;372(24):2285-95.

5. Campbell BC, Mitchell PJ, Kleinig TJ, et al. Endovascular therapy for ischemic stroke with perfusion-imaging selection. N Engl J Med. 2015;372(11):1009-18.

6. Jovin TG, Chamorro A, Cobo E, et al. Thrombectomy within 8 hours after symptom onset in ischemic stroke. N Engl J Med. 2015;372(24):2296-306.
7. Adeoye O, Albright KC, Carr BG, et al. Geographic access to acute stroke care in the United States. Stroke. 2014;45(10):3019-24.

8. Schlemm E, Ebinger M, Nolte CH, Endres M, Schlemm L. Optimal transport destination for ischemic stroke patients with unknown vessel status: use of prehospital triage scores. Stroke. 2017; 48(8):2184-91.

9. Perez de la Ossa N, Carrera D, Gorchs M, et al. Design and validation of a prehospital stroke scale to predict large arterial occlusion: the rapid arterial occlusion evaluation scale. Stroke. 2014;45(1):87-91.

10. Katz BS, McMullan JT, Sucharew H, Adeoye O, Broderick JP. Design and validation of a prehospital scale to predict stroke severity: Cincinnati prehospital stroke severity scale. Stroke. 2015;46(6):1508-12.

11. Kothari R, Hall K, Brott T, Broderick J. Early stroke recognition: developing an out-of-hospital NIH stroke scale. Acad Emerg Med. 1997;4(10):986-90.

12. Kothari RU, Pancioli A, Liu T, Brott T, Broderick J. Cincinnati prehospital stroke scale: reproducibility and validity. Ann Emerg Med. 1999;33(4):373-78.

13. Oostema JA, Konen J, Chassee T, Nasiri M, Reeves MJ. Clinical predictors of accurate prehospital stroke recognition. Stroke. 2015;46(6):1513-17.

14. Scheitz JF, Abdul-Rahim AH, MacIsaac RL, et al. Clinical selection strategies to identify ischemic stroke patients with large anterior vessel occlusion: results from SITS-ISTR (Safe Implementation of Thrombolysis in Stroke International Stroke Thrombolysis Registry). Stroke. 2017;48(2):290-97.

15. Richards CT, Huebinger R, Tataris KL, et al. Cincinnati prehospital stroke scale can identify large vessel occlusion stroke. Prehosp Emerg Care. 2018;22(3):312-18.

16. Mohamad NF, Hastrup S, Rasmussen M, et al. Bypassing primary stroke centre reduces delay and improves outcomes for patients with large vessel occlusion. Eur Stroke J. 2016;1(2): 85-92.

17. Meretoja A, Keshtkaran M, Tatlisumak T, Donnan GA, Churilov L. Endovascular therapy for ischemic stroke: save a minute-save a week. Neurology. 2017;88(22):2123-27. 